\title{
Research Paper \\ Comparison of the Effect of Music Therapy and Writing Therapy on the Anxiety and Depression of Students with Cancer
}

\author{
Parisa Naseri ${ }^{1}$ (D), Mahmoud Shirazi ${ }^{2, *}$ (D), Gholamreza Sanagouye Moharer ${ }^{3}$ (iD) \\ ${ }^{1}$ PhD Student, Department of Psychology, Zahedan Branch, Islamic Azad University, Zahedan, Iran \\ 2 Associate Professor, Department of Psychology, University of Sistan and Baluchestan, Sistan and Baluchestan, Iran \\ ${ }^{3}$ Assistant Professor, Department of Psychology, Zahedan Branch, Islamic Azad University, Zahedan, Iran \\ * Corresponding author: Mahmoud Shirazi, Associate Professor, Department of Psychology, University of Sistan and Baluchestan, \\ Sistan and Baluchestan, Iran. E-mail: Mshirazi@edpsy.usb.ac.ir \\ How to Cite this Article: \\ Naseri P, Shirazi M, Sanagooi Moharr Gh. Comparison of the Effect of Music Therapy and Writing Therapy on the Anxiety and \\ Depression of Students with Cancer. Iran J Rehabil Res Nurs. 2020;7(1):55-65. \\ DOI: 10.29252/ijrn.7.1.55
}

Received: 25 Apr 2020 Accepted: 29 Aug 2020

\section{Keywords:}

Music Therapy

Writing

Anxiety

Depression

Cancer

(C) 2020 Iranian Journal of

Rehabilitation Research in Nursing

\begin{abstract}
Introduction: Depression and anxiety have a negative effect on cancer, so its evaluation and treatment are important in these patients. The aim of this study was to compare the effectiveness of two music-based therapies and to write down the levels of anxiety and depression in students with cancer.

Methods: The present semi-experimental study was a pretest and posttest with a control group. The statistical population consisted of all students aged 14-8 years with cancer in the first six months of 2019 who were hospitalized in Bandar Abbas Children's Hospital; A total of 45 subjects were randomly selected and randomly assigned to two test groups and a control group (15 subjects each). All subjects were tested before and after treatment with the Spence Anxiety Test and the Barlson Depression. The experimental groups underwent music therapy and writing therapy, but the control group did not receive any intervention. The data were analyzed by statistical tests.

Results: The results showed that music therapy and writing therapy had an effect on depression and anxiety in students with cancer, and there was no significant difference between the effectiveness of the two treatments $(P<0.001)$.

Conclusions: Based on the results of the research, music therapy and writing therapy can be used as a complementary, non-pharmacological treatment and effective methods to reduce anxiety and depression in students with cancer.
\end{abstract}

\section{Extended Abstract}

\section{OBJECTIVE}

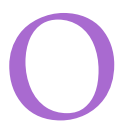

ne of the diseases that can be considered a serious threat to the survival and physical and mental health of children is cancer (1). Cancer is one of the most important health problems in Iran and around the world, and is the third leading cause of death and the second largest group of chronic and noncommunicable diseases (3). Cancers are a disease characterized by uncontrolled cell growth and invasion of systemic localized tissues (4). And according to a study conducted in the Iranian population, the death rate from cancer is estimated to be about $4 \%$ in children fewer than 5 years of age and about $13 \%$ in children aged
5 to 15 years (7). The process of diagnosis, treatment, and complications of the disease necessitates hospitalization of the child for long periods of time, which is an unpleasant, irritating, and frightening experience, and puts children under stress. In addition, repeated hospitalizations separate children from family, school, and the social environment, creating depression, anxiety, stress, and feelings of helplessness (8). Therefore, the study of depression and anxiety is an important variable in the field of cancer that needs to be considered in the psychosocial care of these children in the treatment process (14). On the other hand, cancer 
and its treatment have various dimensions, so in addition to clinical issues, it is necessary to pay attention to complementary and non-pharmacological treatments (15). In this regard, non-pharmacological treatments have attracted the attention of various patients and are used as a supplement in acute cases along with drug therapies as complementary therapies. Used, these therapies include music therapy and writing therapy, etc., due to the ease of the correct way to write and do it automatically without face-to-face visits, as well as the less intervention of the therapist in the treatment process and its easy implementation in the short term. Other treatment methods that require long face-to-face and continuous sessions are differentiated (32). Therefore, the use of two non-pharmacological treatments and the comparison of these three methods in terms of affecting anxiety and depression in cancer patients is an innovative aspect of the present study and although the effectiveness of any of the treatment methods offered to the cancer community is limited; Therefore, due to the lack of research gap in this field, the aim of this study was to compare the effect of music therapy and writing therapy on anxiety and depression in students with cancer.

\section{MATERIALS AND METHODS}

The present semi-experimental study was a pretest and posttest with a control group. The statistical population consisted of all students aged 8-14 years with cancer in the first six months of 2019 who were hospitalized in Bandar Abbas Children's Hospital; A total of 45 subjects were randomly selected and randomly assigned to two test groups and a control group (15 subjects each). All subjects were tested before and after treatment with the Spence Anxiety Test and the Barlson Depression. The experimental groups underwent music therapy and writing therapy, but the control group did not receive any intervention. The data were analyzed by statistical tests.

\section{RESULTS}

The results showed that music therapy and writing therapy had an effect on depression and anxiety in students with cancer, and there was no significant difference between the effectiveness of the two treatments $(\mathrm{P}<0.001)$.

Table 1.

\begin{tabular}{lcccccc}
\hline Variable & \multicolumn{2}{c}{ Pre-Test } & \multicolumn{2}{c}{ After Test } & \multicolumn{2}{c}{ Post-Test Comparison } \\
\cline { 2 - 6 } & $\mathbf{M}$ & SD & M & SD & F & P \\
\hline Anxiety & 64.4 & 16.4 & 63.8 & 16 & 0.001 \\
$\quad$ Control & 59.01 & 16.7 & 36.3 & 13.2 & & \\
Music therapy & 52.93 & 22.62 & 33 & 10.8 & & \\
$\quad$ Writing therapy & & & & & 35.390 & 0.001 \\
Depression & 44.8 & 4.2 & 44.2 & 3.8 & & \\
$\quad$ Control & 48.4 & 3.9 & 42 & 6.7 & & \\
$\quad$ Music therapy & 47.2 & 3.5 & 44.7 & 4.1 & & \\
$\quad$ Writing therapy & & & & & \\
\hline
\end{tabular}

\section{CONCLUSION}

Based on the results of the research, music therapy and writing therapy can be used as a complementary, nonpharmacological treatment and effective methods to reduce anxiety and depression in students with cancer.

\section{Ethical Considerations}

The authors state that all relevant ethical principles have been observed in this study, including the confidentiality of the questionnaires, the informed consent of the participants in the research, and the authority to withdraw from the research. In addition, this article is taken from the doctoral dissertation of Psychology, Islamic Azad University, Zahedan Branch, with the number $5 / 7773$ dated $08 / 10 / 2018$. And it has been approved by the ethics committee of Bandar Abbas University of Sciences under the code Ir.HUMS.REC.1398.255

\section{Funding or Supports}

This research was conducted without any financial support.

\section{Authors Contribution}

Ms. Parisa Naseri was in charge of the initial writing and preparation of the initial draft of the article. Dr. Gholamreza Sanagavi was in charge of statistical analysis. Dr. Mahmoud Shirazi has submitted, followed and reviewed the article.

\section{Conflict of Interest}

The authors state that there is no conflict of interest between the authors in this study.

\section{Applicable Remarks}

Based on the results of the present study, the use of music-based therapy and writing can be used as a means to relieve anxiety and depression in patients with cancer, so its use in the agenda of medical centers, hospitals, Health services, counseling and psychotherapy and families should be considered and gender and cultural differences should also be considered.

\section{Acknowledgments}

We would like to thank all the patients and staff of the oncology department of Bandar Abbas Children's Hospital and all those who have helped us in this research. 


\section{مقايسه اثربخشى دو درمان مبتنى بر موسيقى و نوشتن بر ميزان اضطراب و افسردىى دانش آموزان مبتلا به سرطان}

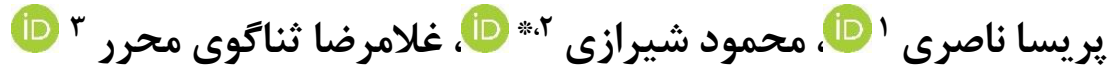

' دانشجوى دكترى تخصصى، كروه روانشناسى، واحد زاهدان، دانشكاه آزاد اسلامى، زاهدان، ايران

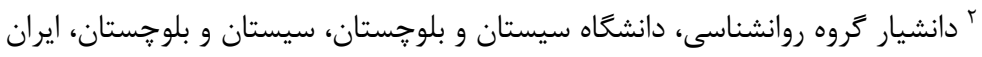

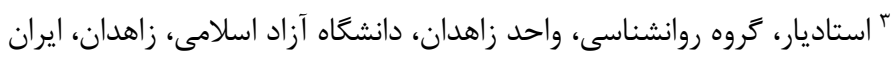

" نويسنده مسئول: محمود شيرازى، دانشيار گروه روانشناسى، دانشگاه سيستان و بلوخستان، سيستان و بلوجستان، ايران. ايميل:Mshirazi@edpsy.usb.ac.ir

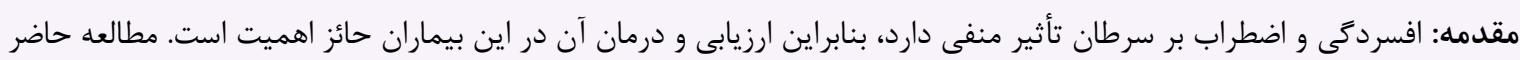

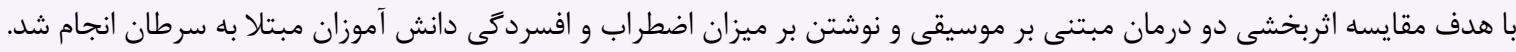

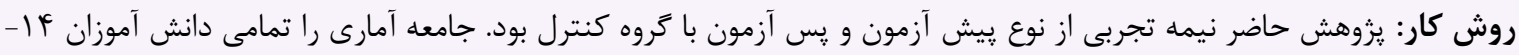

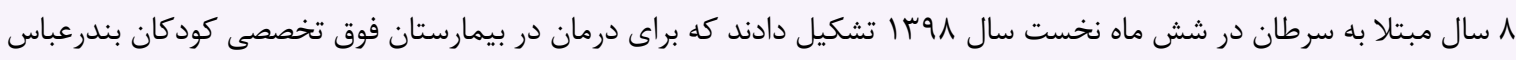

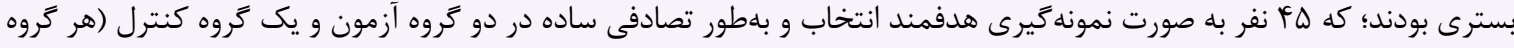

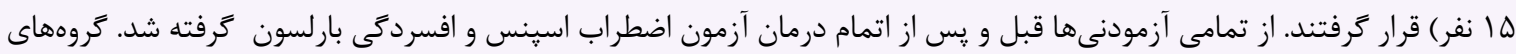

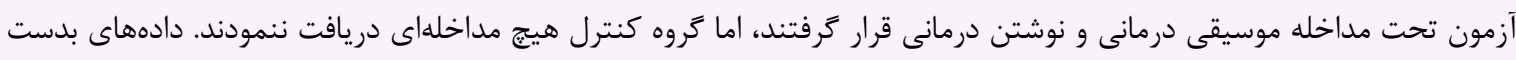

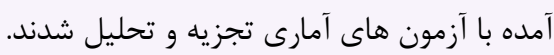

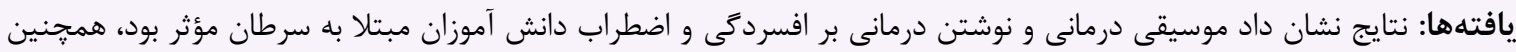

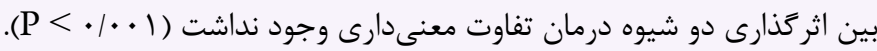

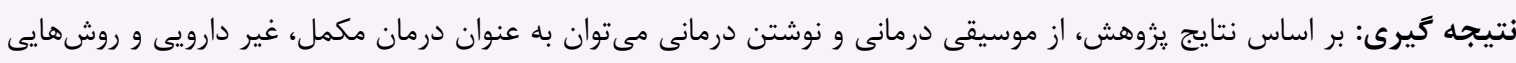

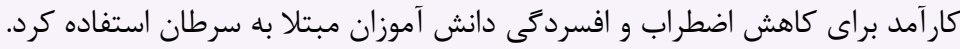

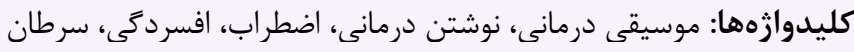

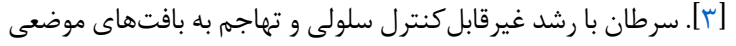

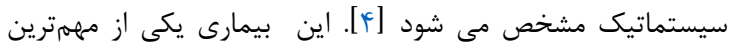

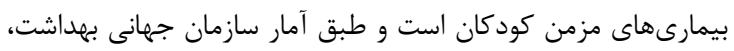

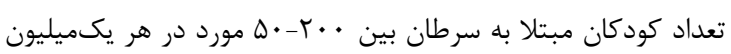

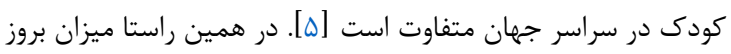

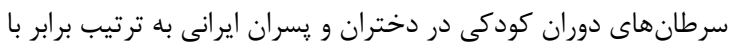

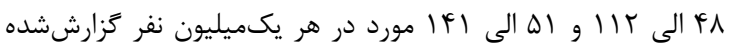

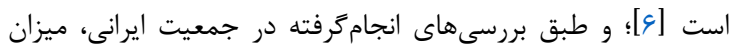

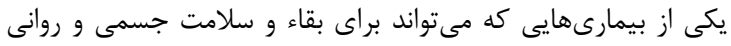

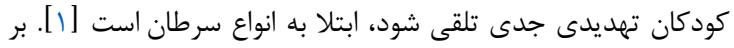

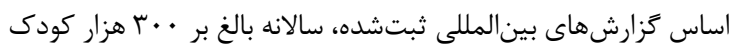

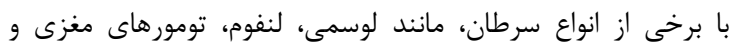

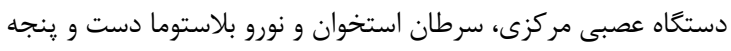

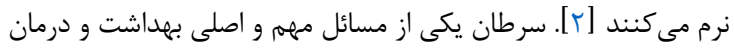

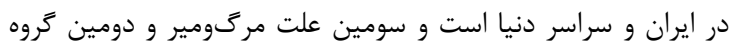

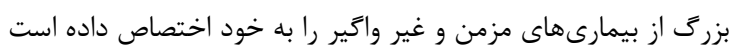


افزايش داشته است. مطالعات مختلف نشان دادهاند كه نوشتن در مورد

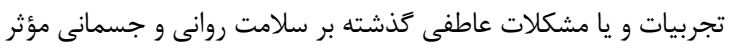

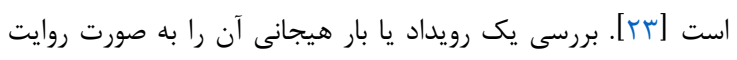

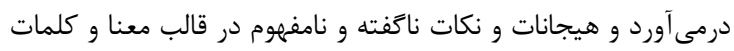

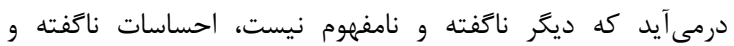

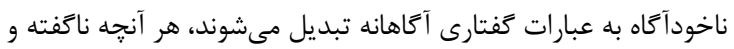

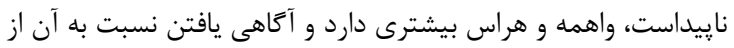

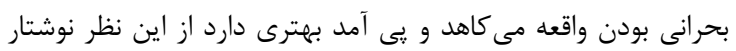

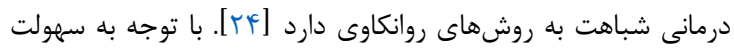

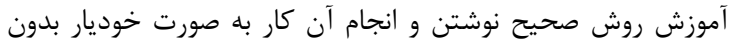

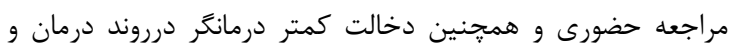

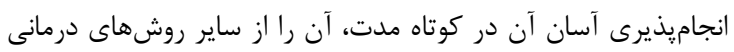

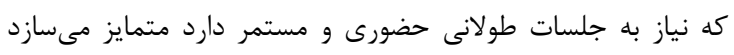

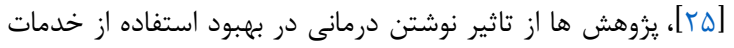

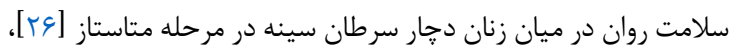

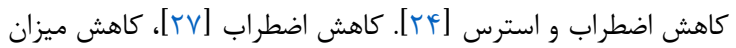

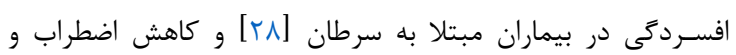

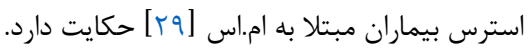

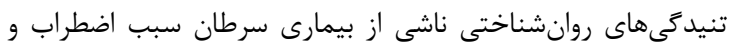

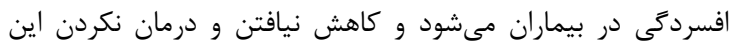

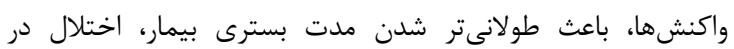

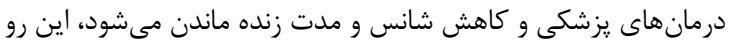

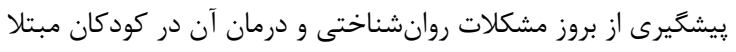

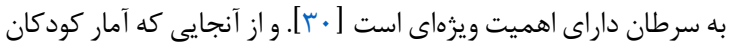

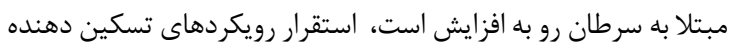

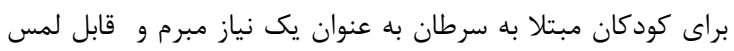

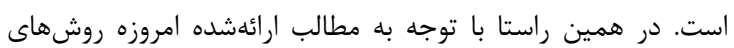

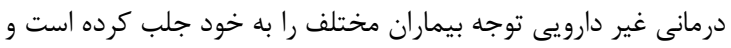

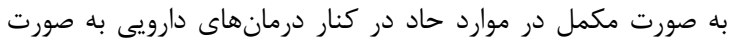

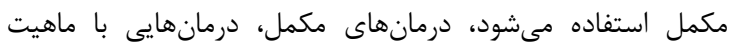

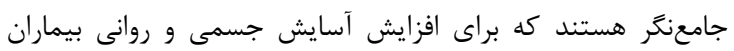

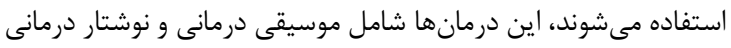

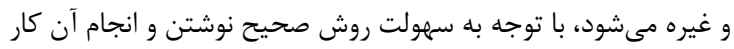

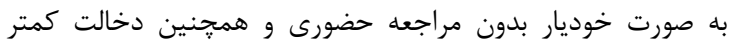

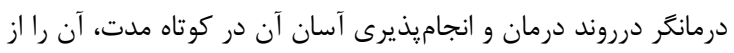

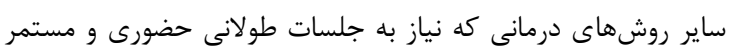
[دارد متمايز مى سازد [ [ابـ]

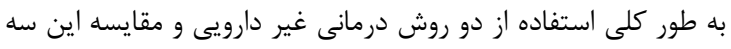

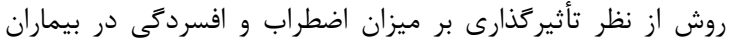

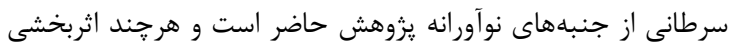

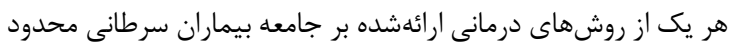

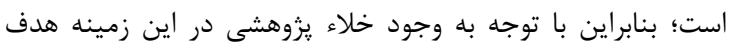

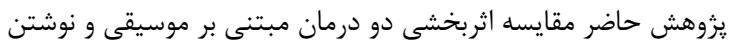

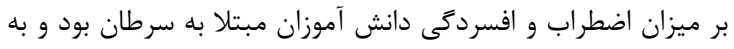

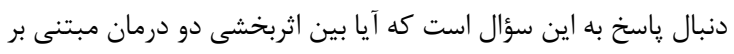

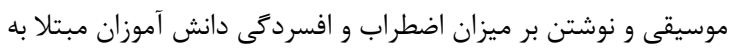

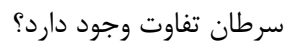

مركَومير ناشى از سرطان، در كودكان زير ه سال، حدود ؟ أ درصد و

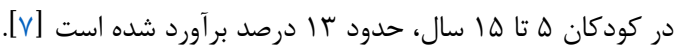

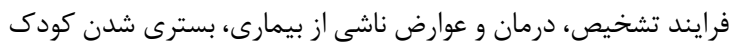

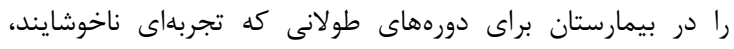

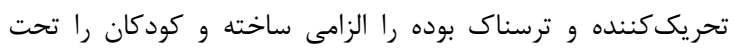

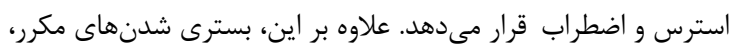

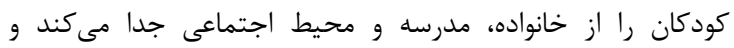

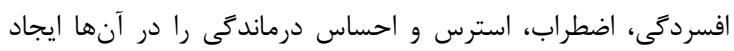

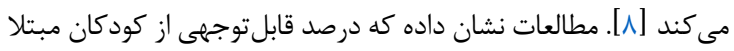

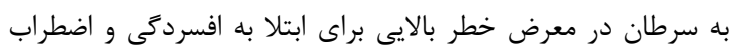

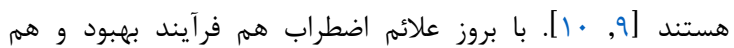

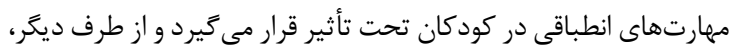

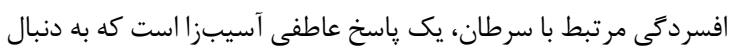

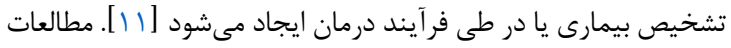

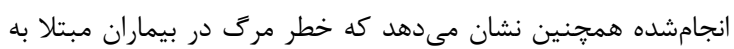

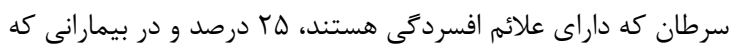

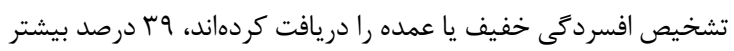

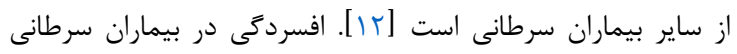

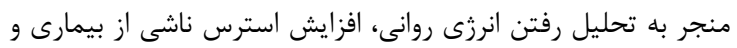

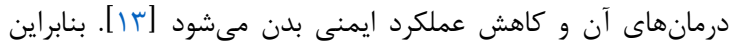

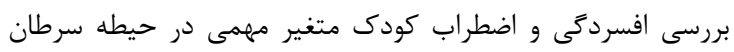

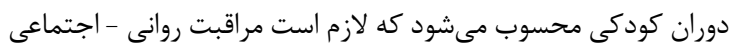

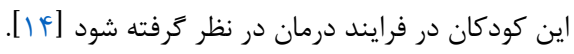

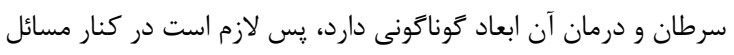

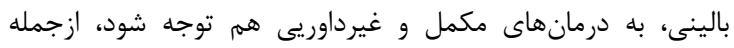

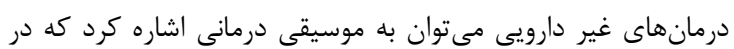

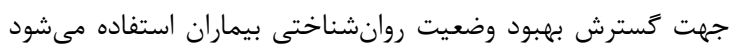

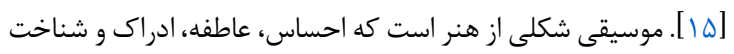

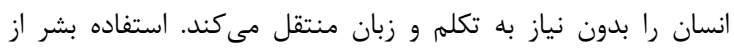

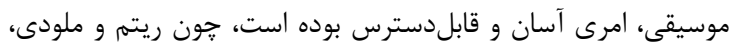

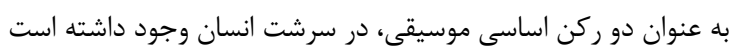

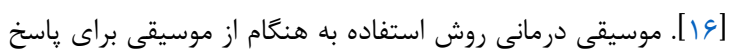

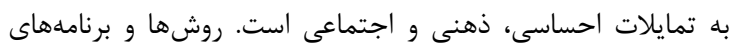

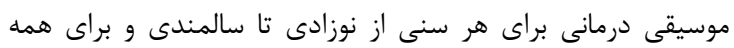

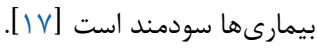

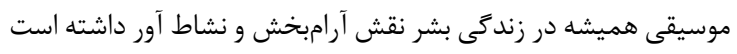

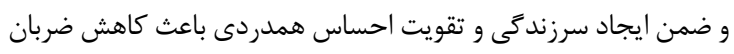

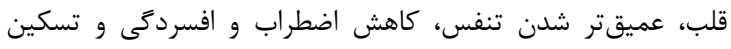

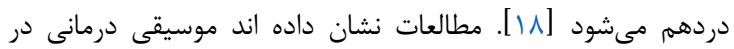

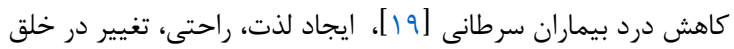

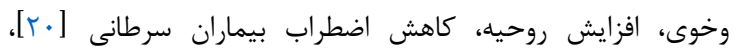

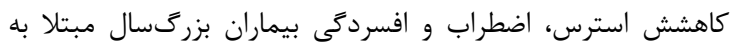

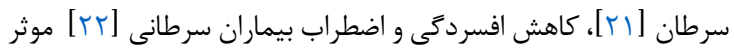

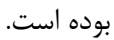

همجنين تحقيقات نشان دادهاند كه نوشتن و برون ريزى احساسات

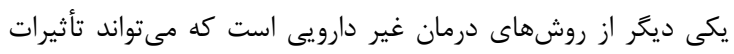

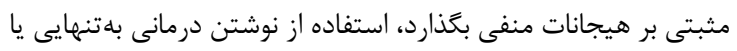

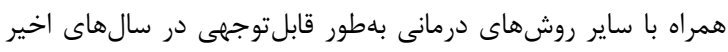


محتوى :رسش نامه توسط اساتيد دانشكده يرستارى و مامائى قائن

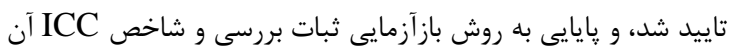

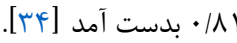

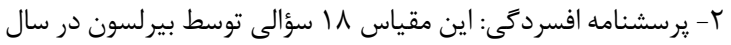

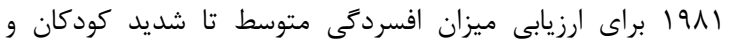

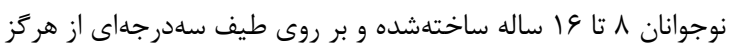

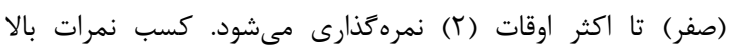

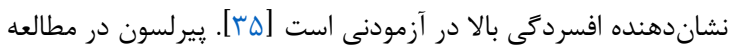

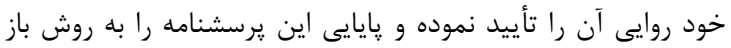

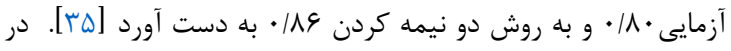

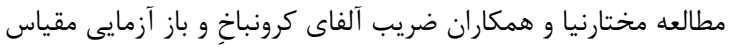

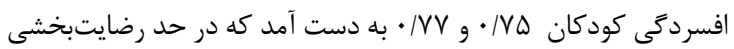

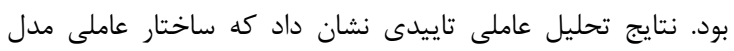

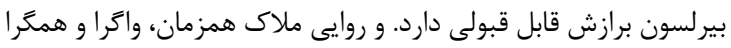

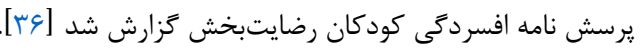

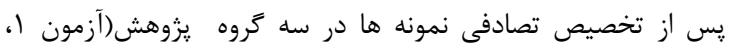

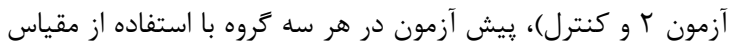

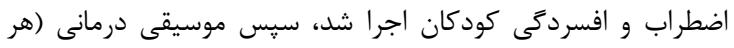

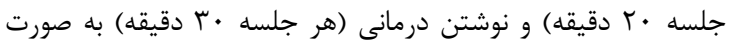

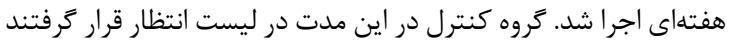

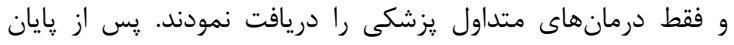

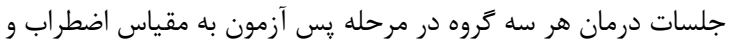
افسردگى كودكان مجدد ياسخ دادند روند جلسات آموزش موسيقى درمانى: در مرديخ دروه دادند موسيقى درمانى از

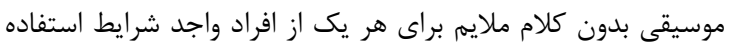

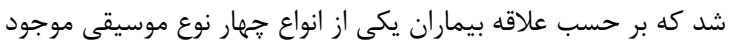
در جعبه موسيقى (كه توسط متخصصين انجمن موسيقى ايران

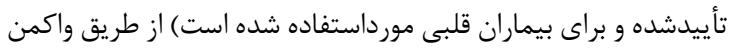

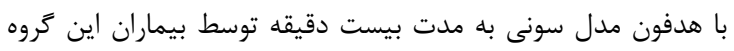

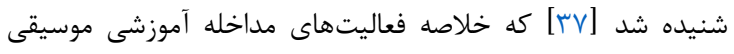

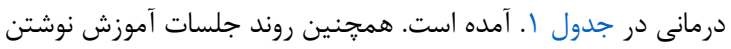

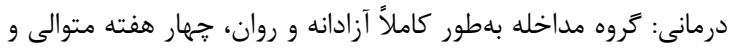

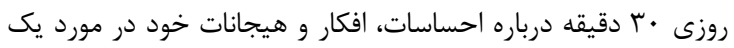

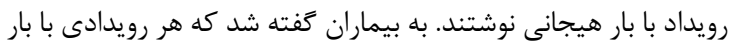

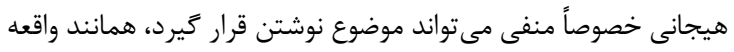

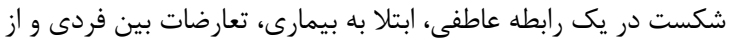

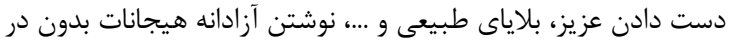

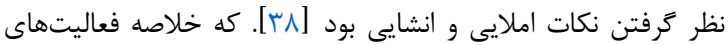

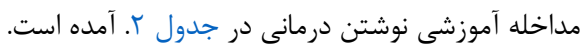

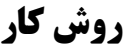

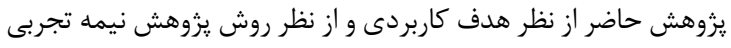

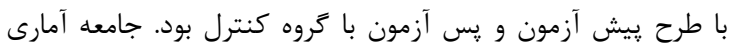

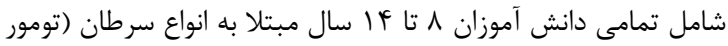

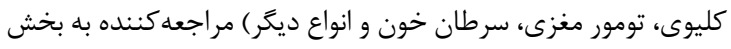

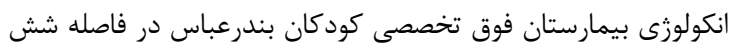

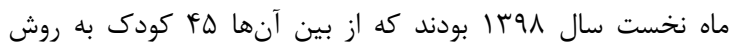

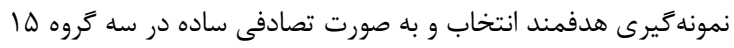

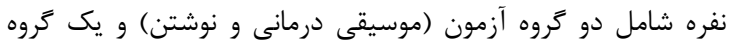

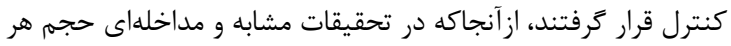

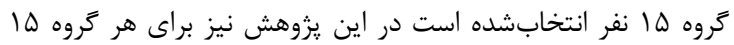

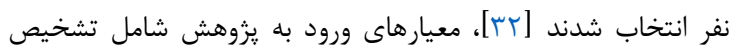

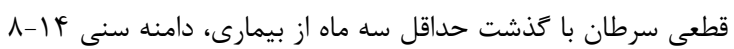

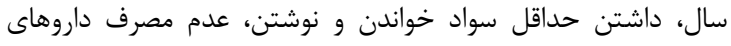

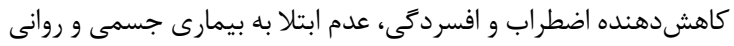

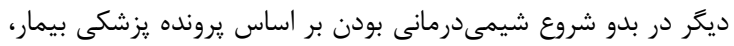

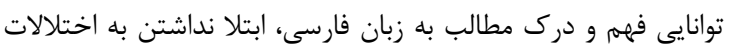

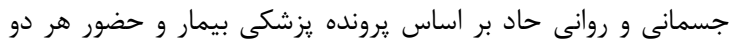

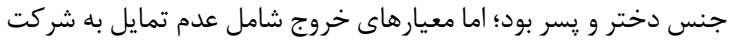

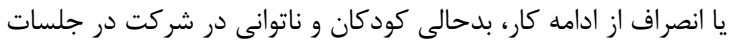

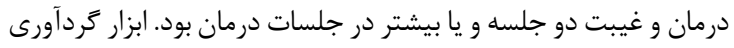

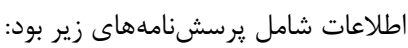

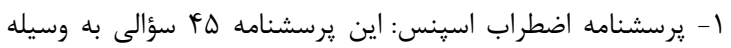

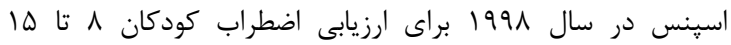

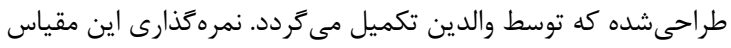

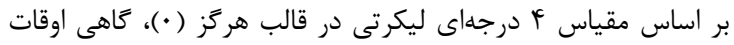

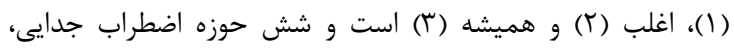

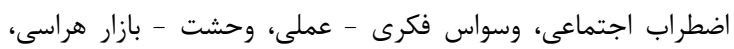

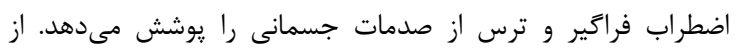

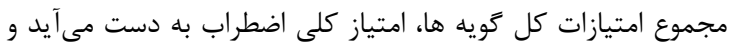

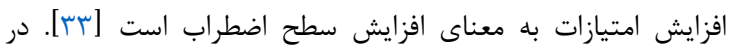

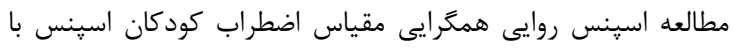

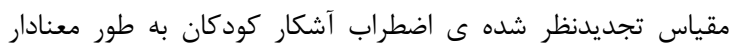

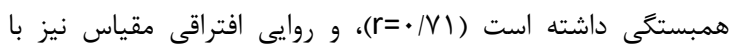

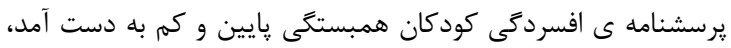

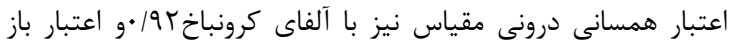

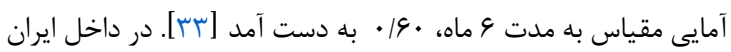

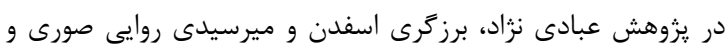

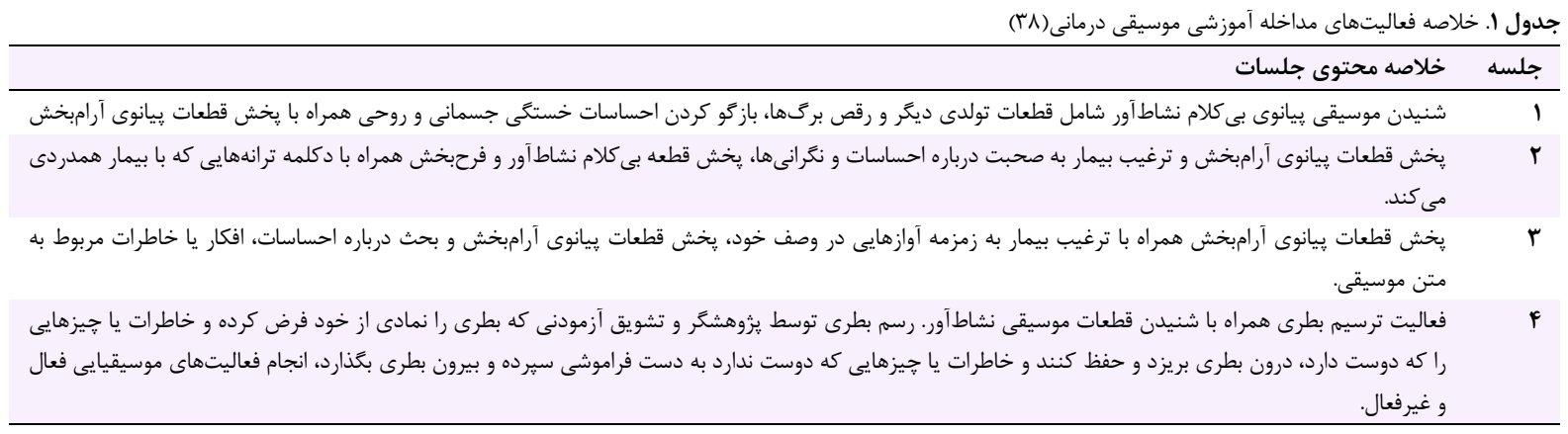


جدول r. خلاصه فعاليتهاى مداخله آموزشى نوشتن درمانى [1/]

\begin{tabular}{|c|c|}
\hline خلاصه محتوى جلسات & جلسه \\
\hline 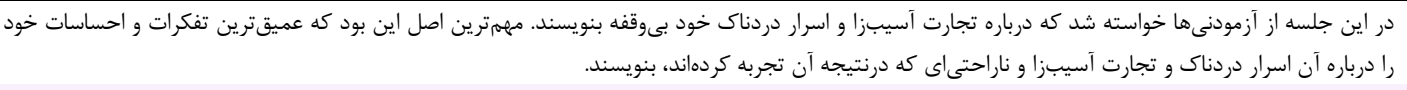 & 1 \\
\hline 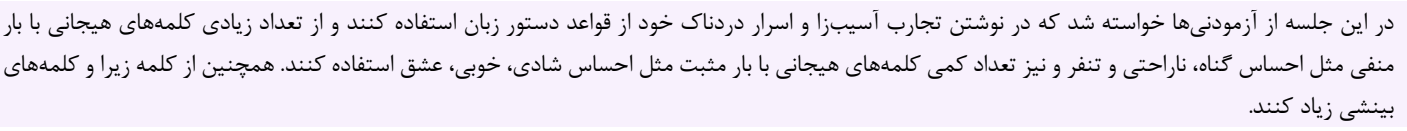 & $r$ \\
\hline 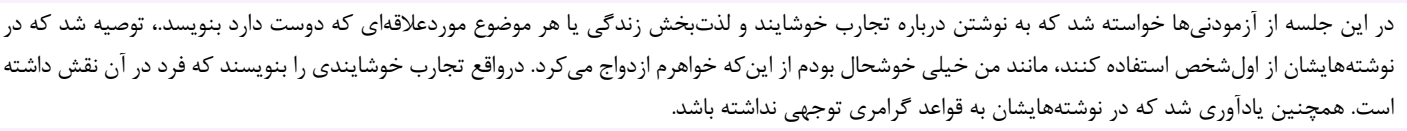 & r \\
\hline 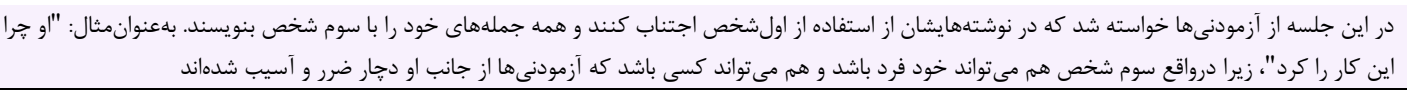 & f \\
\hline
\end{tabular}

\section{يافته ها}

بر اساس يافته هاى توصيفى وضعيت ميانكين و انحراف معيار سن

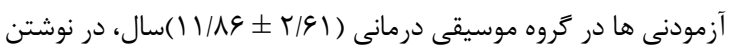
درمانى (1//

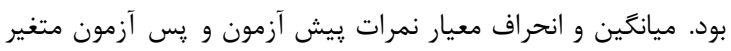

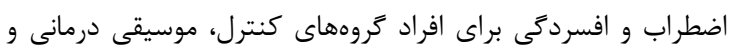

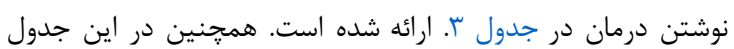

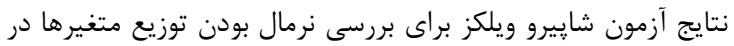

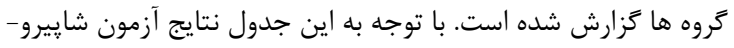

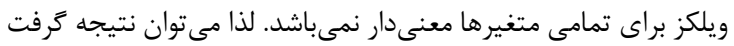

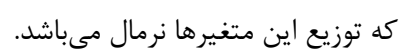

در راستاى رعايت اصول اخلاقى يزوهشى و بلمنظور حفظ حقوق

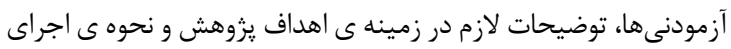

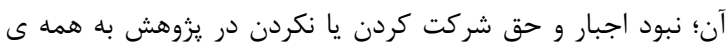

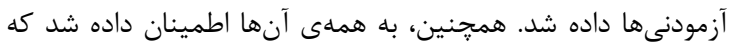

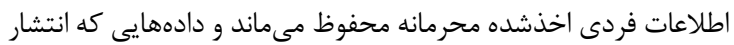

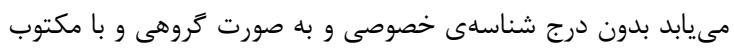

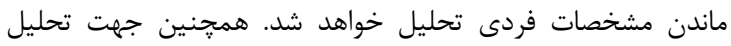

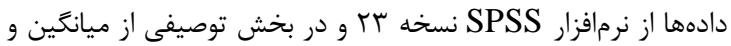

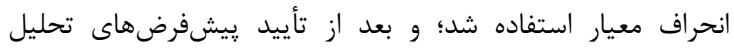

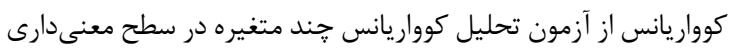

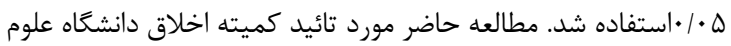

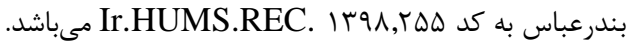

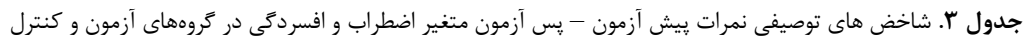

\begin{tabular}{|c|c|c|c|c|}
\hline $\mathbf{P}$ & شاييرو- ويلكز & انحراف معيار & ميانگين & متغير \\
\hline & & & & اضطراب \\
\hline & & & & ل بيش آزمون \\
\hline . /9४४ & $\cdot|\& \Delta|$ & $19 / 4$ & $q 4 / 4$ & كنترل \\
\hline •/ $/ A \vee$ & $1 / \cdot 11$ & $19 / \mathrm{V}$ & $\Delta 9 / \cdot 1$ & موسيقى درمانى \\
\hline \multirow[t]{2}{*}{ - /TYG } & $1 / \cdot F q$ & Tr/GT & $\Delta r / q r$ & نوشتن درمانى \\
\hline & & & & ع پِ آزمون \\
\hline$\cdot / \Delta \cdot r$ & - /ATS & 19 & $8 \% / 1$ & كنترل \\
\hline$\cdot / 499$ & $\cdot \mid \Lambda \uparrow \Lambda$ & $\mid r / T$ & re/T & موسيقى درمانى \\
\hline \multirow[t]{3}{*}{. /ITV } & I/IVF & $1 \cdot 11$ & זr & نوشتن درمانى \\
\hline & & & & افسردَىى \\
\hline & & & & ليش \\
\hline$\cdot / 1 \Delta \wedge$ & $1 / / T V$ & $F / T$ & $F F / \Lambda$ & كنترل \\
\hline . & $1 / . r 9$ & $r / 9$ & FN/F & موسيقى درمانى \\
\hline \multirow[t]{2}{*}{$\cdot / 4 \cdot \Delta$} & - $/ 191$ & $r / \Delta$ & $F V / T$ & نوشتن درمانى \\
\hline & & & & يس آزمون \\
\hline - $/ K Y \Lambda$ & $1 / \cdot r t$ & $r / \Lambda$ & $F \psi / T$ & كنترل \\
\hline$\cdot / \Delta F V$ & $\cdot / \vee \wedge q$ & GIV & et & موسيقى درمانى \\
\hline . /NF & $1 / 10$ & $\kappa / 1$ & $r F / V$ & نوشتن درمانى \\
\hline
\end{tabular}

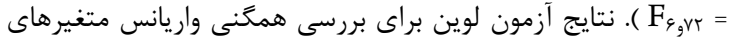

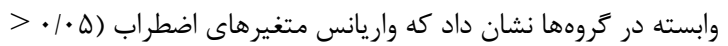
مر ( F

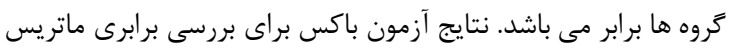

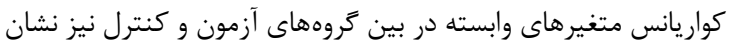

به منظور مقايسه تاثير موسيقى درمانى و نوشتن درمانى بر اضطراب و

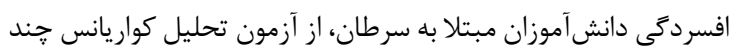

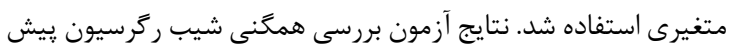

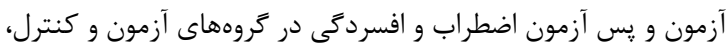

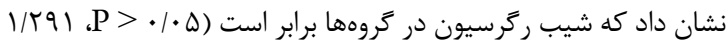




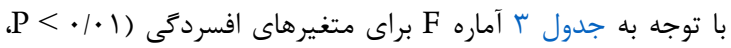

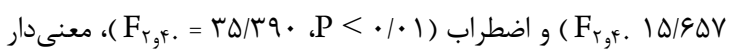

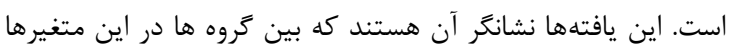

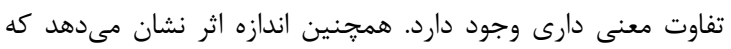

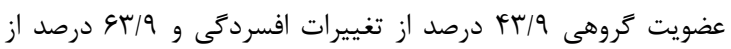

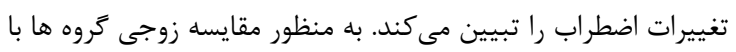

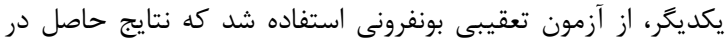
جدول ه. ارائه شده است.
داد كه ماتريس كواريانس متغيرهاى وابسته در زروهها برابر است

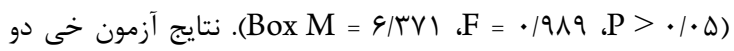

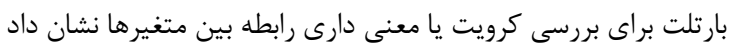

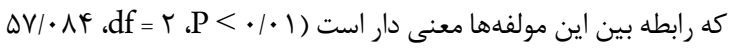

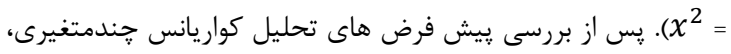

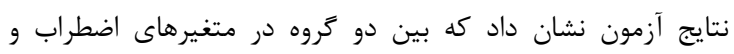
افسرد Wilks Lambda=•/l ar ، F

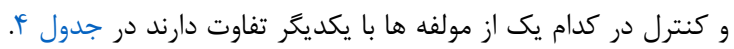

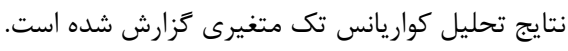

\begin{tabular}{|c|c|c|c|c|c|c|}
\hline \multirow[b]{2}{*}{ 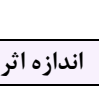 } & \multirow[b]{2}{*}{ سطح معنادارى } & & \multicolumn{4}{|c|}{ جدول F. نتايج تحليل كواريانس تك متغيرى تفاوت كروههاى آزمون و كنترل در متغيرهاى اضطراب و افسردىى } \\
\hline & & $\mathbf{F}$ & ميانگين مجذورات & درجه آزادى & مجموع مجذورات & متغير \\
\hline & & & & & & 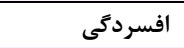 \\
\hline \multirow[t]{3}{*}{. « rq } & 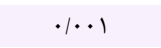 & $10 / 9 \Delta \mathrm{V}$ & rV/Vrq & r & $V \Delta / \& Y \wedge$ & 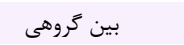 \\
\hline & & & $r / 4)$. & r. & $99 / 419$ & 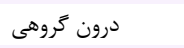 \\
\hline & & & & & & اضطراب \\
\hline \multirow[t]{2}{*}{.1949} & .1 .1 & $r \Delta / r q$. & $|r \Delta / \Delta F|$ & r & $r R I / \cdot A r$ & 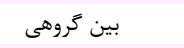 \\
\hline & & & r/Ar. & 4. & $10 \mathrm{H} / 199$ & درون گروهى \\
\hline
\end{tabular}

\begin{tabular}{|c|c|c|c|}
\hline سطح معنى دارى & خطاى استاندارد & اختلاف ميانكين & متغير وابسته \\
\hline & & & اضطراب \\
\hline & & & كنترل \\
\hline $.1 . .1$ & $\cdot / \Delta \wedge T$ & $r q / r+q$ & موسيقى درمانى \\
\hline \multirow[t]{2}{*}{$.1 . .1$} & $\cdot / \Delta \vee \varphi$ & $r \varepsilon / F \Delta \Lambda$ & نوشتن درمانى \\
\hline & & & موسيقى درمانى \\
\hline \multirow[t]{3}{*}{$\cdot 1 \cdot .1$} & $\cdot / \Delta \mathrm{V}$ & $-1 / \vee 91$ & 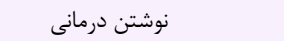 \\
\hline & & & افسردگىى \\
\hline & & & كنترل \\
\hline $.1 . .1$ & $\cdot$ /VHF & $\Delta / \mid V I$ & موسيقى درمانى \\
\hline \multirow[t]{2}{*}{$.1 . .1$} & . /VYS & T/TrI & ن نوشتن درمانى \\
\hline & & & موسيقى درمانى \\
\hline . $/ T Y$ & $\cdot / V / A$ & $-1 / \wedge \Delta$. & لموشت درمانى \\
\hline
\end{tabular}

راحتى، تغيير در خلقوخوى و افزايش روحيه شود و مىتواند اضطراب

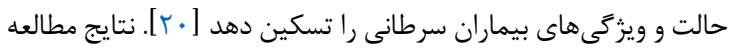

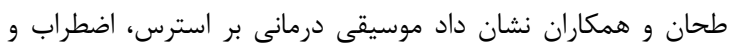

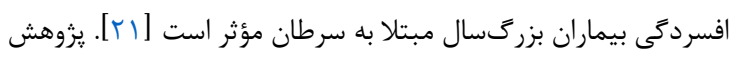
جاسمى و همكاران حاكى از كاهش معنى دار سطح افسرد بـى و اضطراب

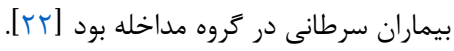

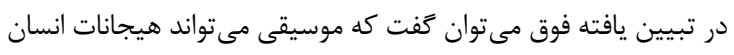

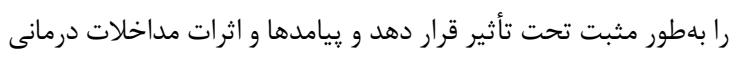

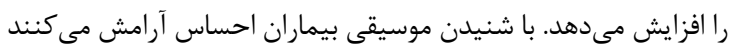

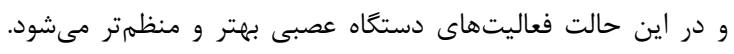
همجنين موسيقى باعث افزايش ثبت امواج آلفاى مغز يا امواج استراحت بـاه

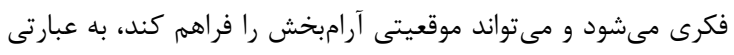

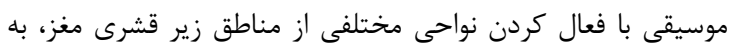

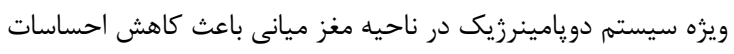

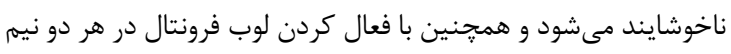

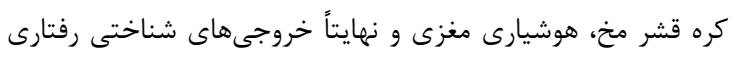

طبق جدول ه نتايج آزمون تعقيبى بونفرونى براى بررسى ميانگَينها

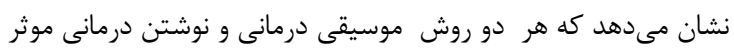

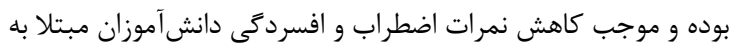

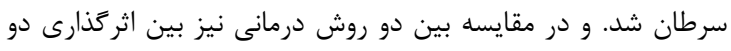
شيوه درمان تفاوت معنى دارى وجود نداشت.

هدف مطالعه حاضر مقايسه اثربخشى دو درمان مبتنى بر موسيقى و

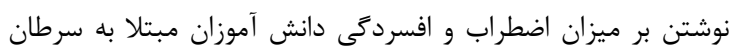

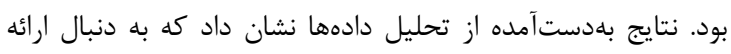

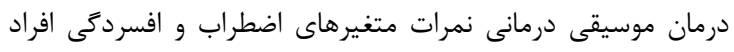

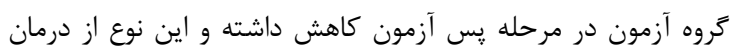

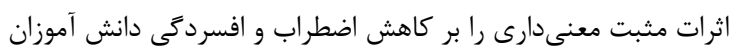

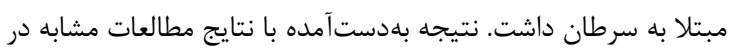

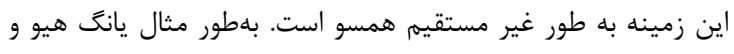

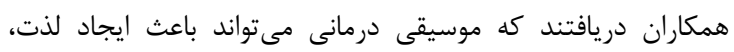


شديد و منفى به فرد فرصت مىدهد تا نسبت به خودآكاهى ييدا كند

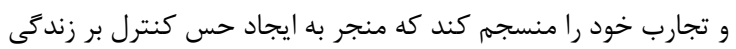

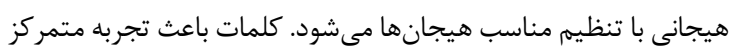

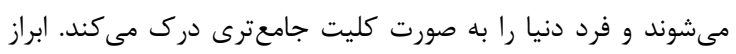

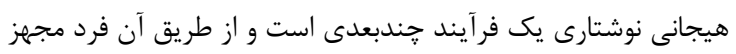

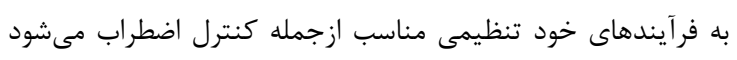

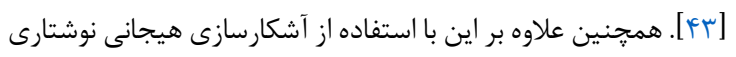

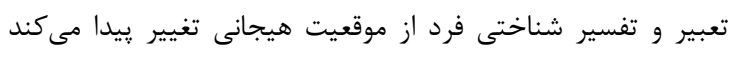

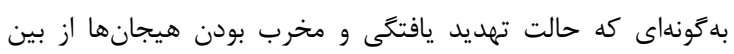

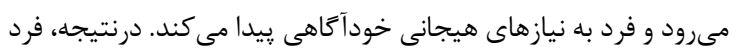

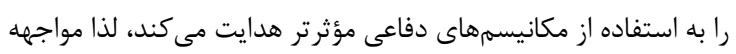

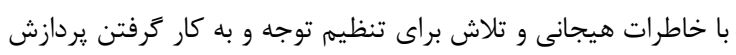

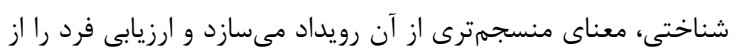

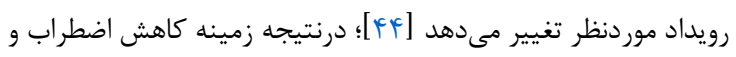

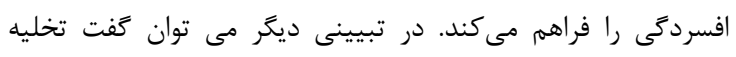

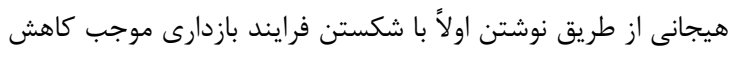

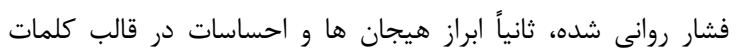

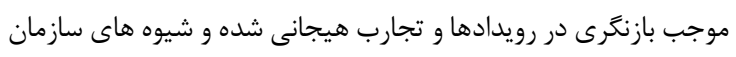

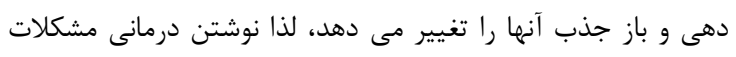

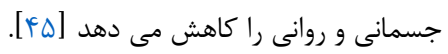

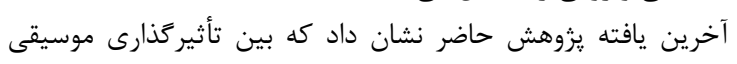

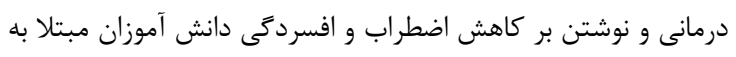

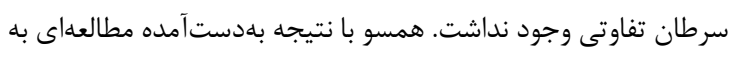

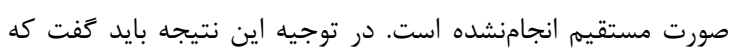

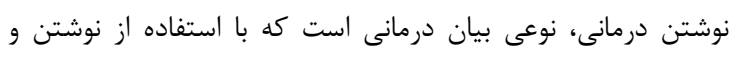

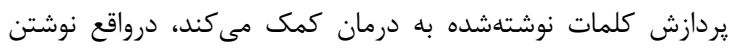

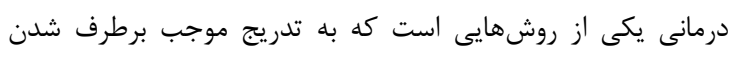

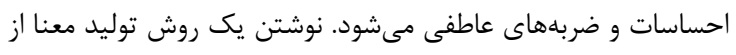

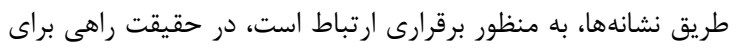

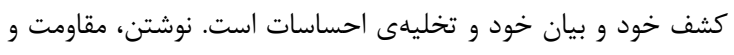

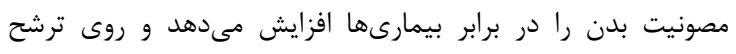

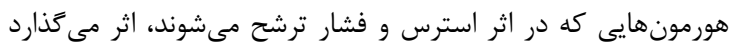

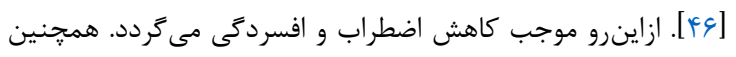

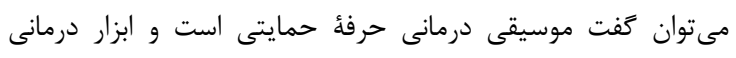

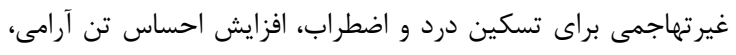

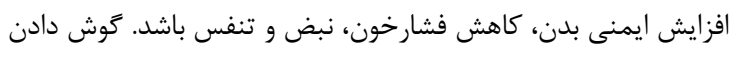

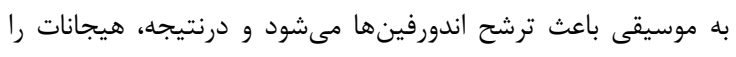

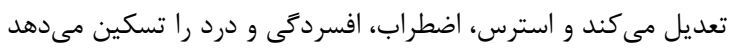

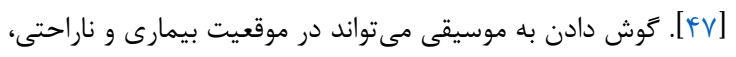
آسايش و راحتى فرد را ارتقاء دهد.

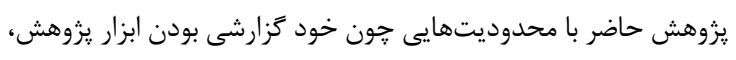

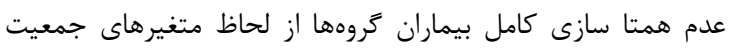

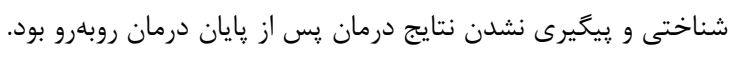

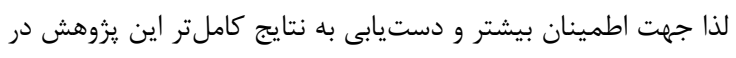

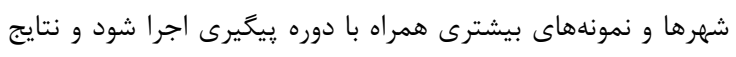

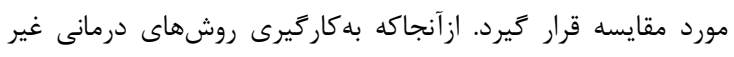

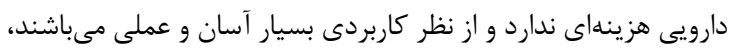

مطلوب را افزايش مى دهد [إr]؛ بنابراين، استفاده از موسيقى درمانى

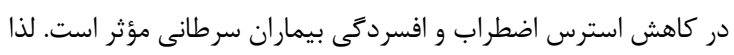

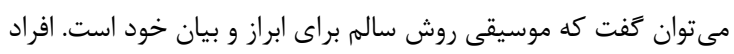

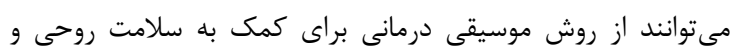

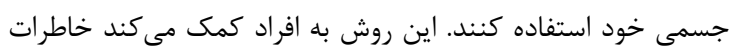

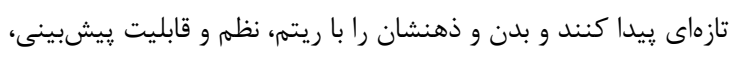

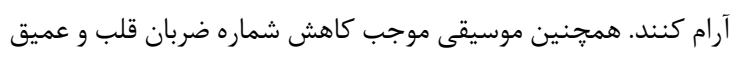

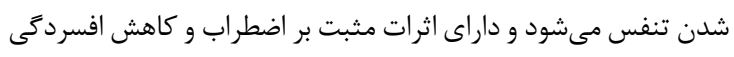

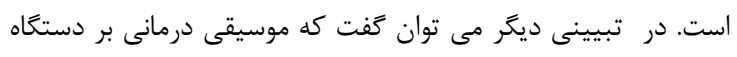

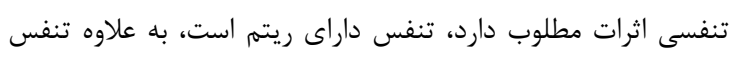

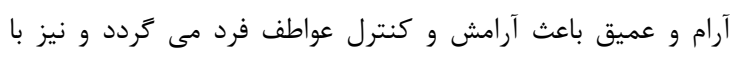

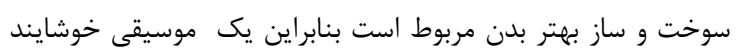

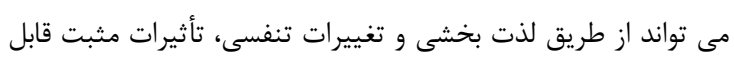

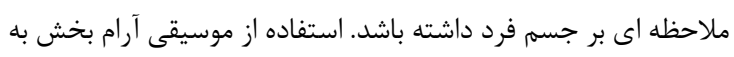

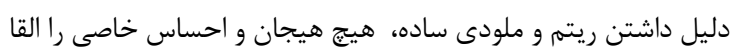

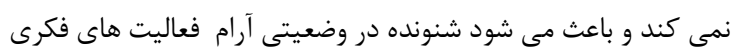

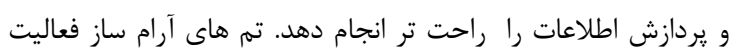

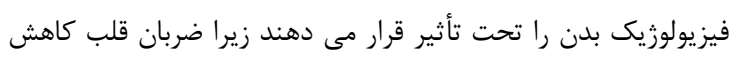

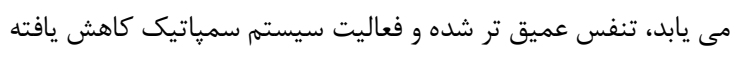

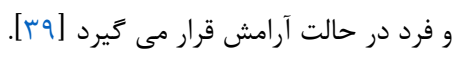

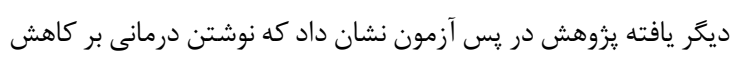

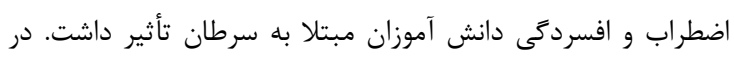

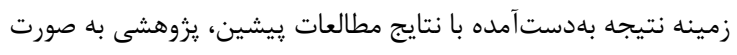

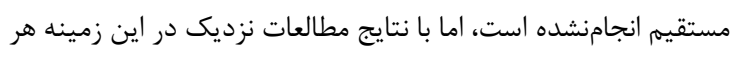

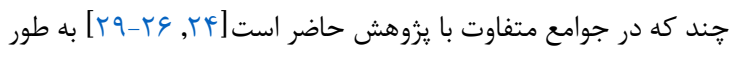
غير مستقيم همسو است. بهعنوانمثال در مطالعهاى موشر و همكاران

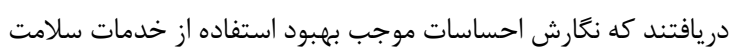

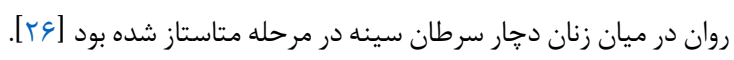

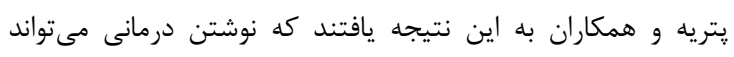
اضطراب و استرس را كاهش دهد [YF]. مطالعه كَودمن و همكاران

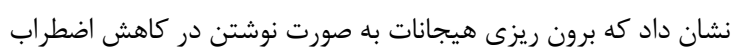

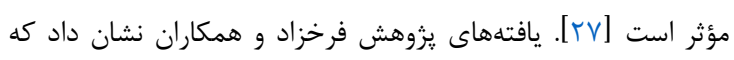

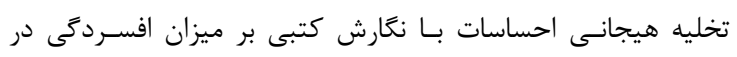

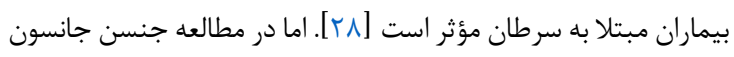

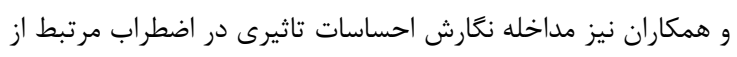

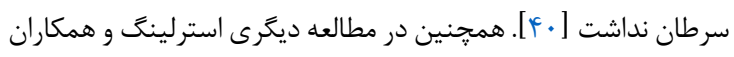

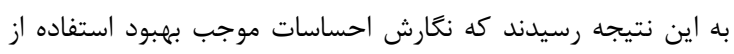

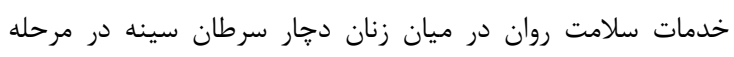

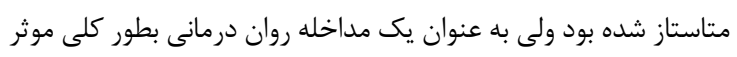

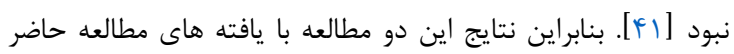

ناهمسو بودند. در تبيين يافته فوق مى توان كفت در فرآيند آشكار سازى هيجانى

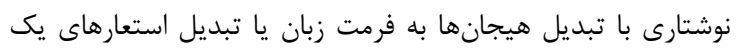

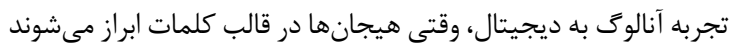

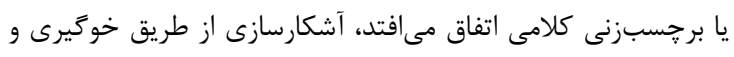

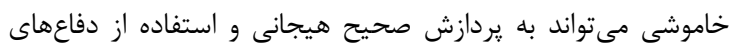

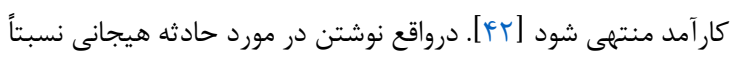




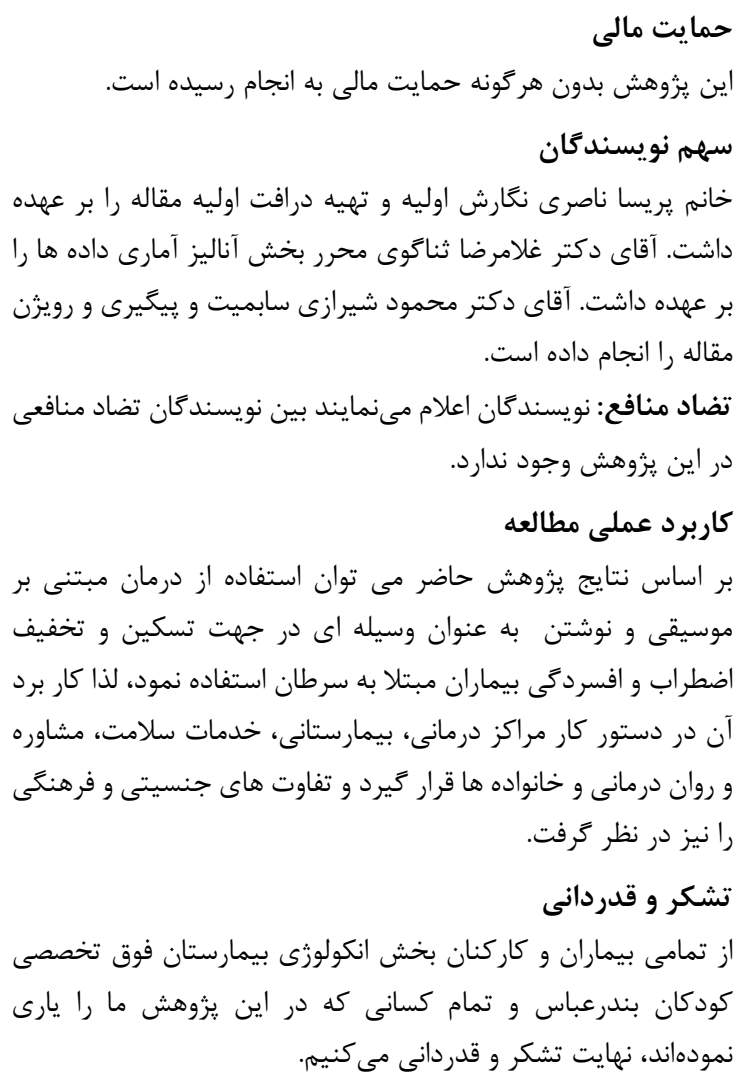

\section{References}

1. Phillips SM, Padgett LS, Leisenring WM, Stratton KK, Bishop K, Krull KR, et al. Survivors of childhood cancer in the United States: prevalence and burden of morbidity. Cancer Epidemiol Biomarkers Prev. 2015;24(4):653-63. doi: 10.1158/1055-9965.EPI-14-1418 pmid: 25834148

2. Steliarova-Foucher E, Colombet M, Ries LA, Moreno F, Dolya A, Bray F. International incidence of childhood cancer, 2001-10: a population-based registry study. Lancet Oncol. 2017;18(6):719-31.

3. Safaeian Z, Hejazi SS, Delavar E, Hoseini Azizi T, Haresabadi M. The Relationship between Caregiver Burden, and Depression, Anxiety and Stress in Family Caregivers of Cancer Patients Referred to Imam Reza Hospital in Bojnurd City [Persian]. JNE. 2017;5(3):7-14. doi: $10.21859 /$ ijpn-05032

4. Mehta S, Lo Cascio C. Developmentally regulated signaling pathways in glioma invasion. Cell Mol Life Sci. 2018;75(3):385-402. doi: 10.1007/s00018-017-2608-8 pmid: 28821904

5. Wiener L, Kazak AE, Noll RB, Patenaude AF, Kupst MJ. Standards for the Psychosocial Care of Children With Cancer and Their Families: An Introduction to the Special Issue. Pediatr Blood Cancer. 2015;62 Suppl 5:S419-24. doi: $10.1002 /$ pbc. 25675 pmid: 26397836

6. Toghian Chaharsoughi N. Legacy- making in palliative care of pediatric cancer. [Persian]. IJCA. 2020;1(4):10-6.

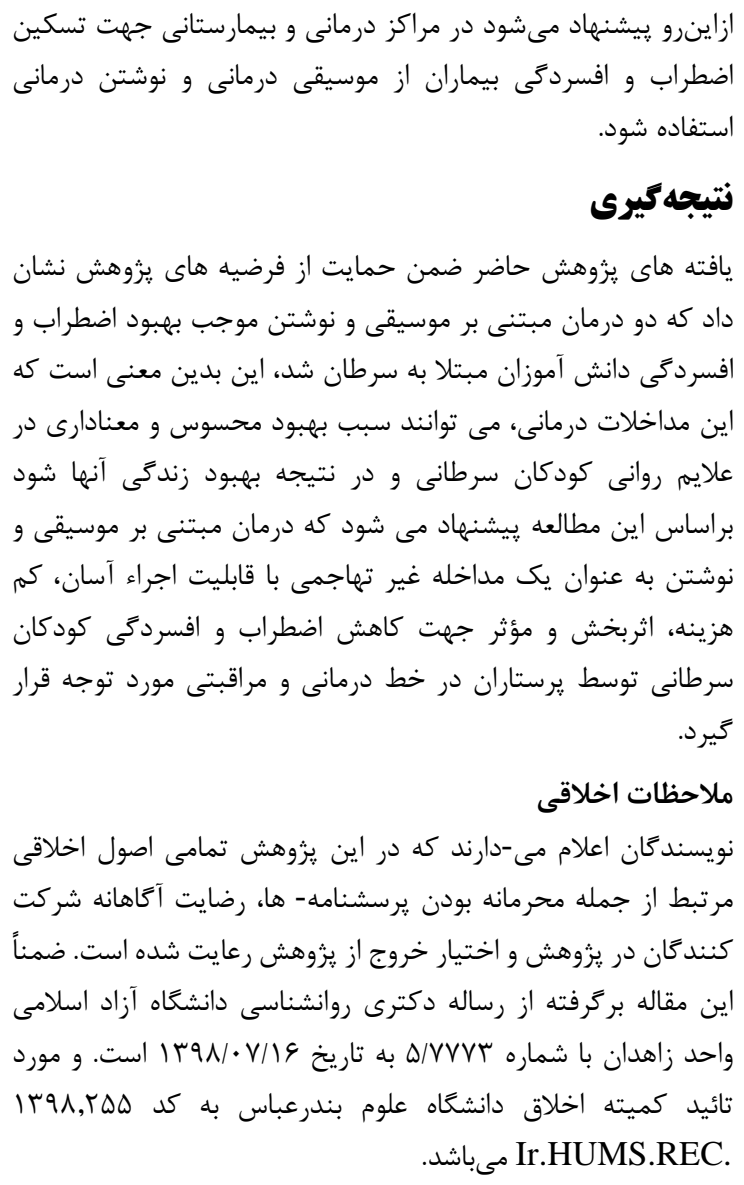

7. Rajabi S, Hashemi J, Pezeshk S. Developing and Assessing the Effectiveness of Cognitive-Behavioral Programs on Body Image in Children with Cancer. [Persian]. MEJDS. 2014;4(3):28-35.

8. Harper FW, Peterson AM, Albrecht TL, Taub JW, Phipps $S$, Penner LA. Satisfaction with support versus size of network: differential effects of social support on psychological distress in parents of pediatric cancer patients. Psychooncology. 2016;25(5):551-8. doi: 10.1002/pon.3863 pmid: 27092714

9. Kim DH, Im YJ. The influence of family management style on psychosocial problems of childhood cancer survivors in Korea. Eur J Oncol Nurs. 2015;19(2):107-12. doi: 10.1016/j.ejon.2014.10.010 pmid: 25466826

10. Muglia-Wechsler A, Bragado-Álvarez C, HernándezLloreda MJ. Eficacia de intervenciones psicológicas dirigidas a promover el ajuste de niños con cáncer y de sus padres: una revisión. Anales de Psicología. 2014;30(1):93-103. doi: 10.6018/analesps.30.1.149161

11. Li M, Kennedy EB, Byrne N, Gerin-Lajoie C, Katz MR, Keshavarz H, et al. Management of Depression in Patients With Cancer: A Clinical Practice Guideline. J Oncol Pract. 2016;12(8):747-56. doi: 10.1200/JOP.2016.011072 pmid: 27382000

12. Kaul S, Avila JC, Mutambudzi M, Russell H, Kirchhoff AC, Schwartz CL. Mental distress and health care use 
among survivors of adolescent and young adult cancer: A cross-sectional analysis of the National Health Interview Survey. Cancer. 2017;123(5):869-78. doi: 10.1002/cncr.30417 pmid: 27859009

13. Fann JR, Thomas-Rich AM, Katon WJ, Cowley D, Pepping M, McGregor BA, et al. Major depression after breast cancer: a review of epidemiology and treatment. Gen Hosp Psychiatry. 2008;30(2):112-26. doi: 10.1016/j.genhosppsych.2007.10.008 pmid: 18291293

14. Shojaei Z, Golparvar M, Aghaei A, Bordbar MR. Comparing the Effectiveness of Group Story Therapy and the Art-Play Therapy on Anxiety and Depression in Children with Cancer: Based on the Framework, Principles and Rules of Cognitive-Behavioral Approach. [Persian]. IJRN. 2019;6(1):50-9.

15. Chen X, Leith H, Aarø LE, Manger T, Gold C. Music therapy for improving mental health in offenders: protocol for a systematic review and meta-analysis. ArXiv Preprint ArXiv. 2015.

16. Sheibani Tazraji F, Pakdaman S, Dadkhah A, Hasanzadeh Tavakoli MR. The Effect of Music Therapy on Depression and Loneliness in Old People. [Persian]. Salmand. 2010;5(2):0.

17. Senobar L. Comparison of the effectiveness of two techniques (Progressive muscle relaxation and music therapy on the rate of fatigue in cancer patients), [MSc. thesis]. [Persian]. Ardabil: University of Mohaghegh Ardabili; 2013.

18. YousefinejadOstadkelayeh A, Madadi A, Majedzadeh S, Shabannia R, Sadeghian N, Zarinara A. The effect of music therapy on chronic pain in patients with cancer. [Persian]. J Qazvin Univ Med Sci. 2005;9(1):39-42.

19. Elliott M, Worrall-Carter L, Page K. Factors contributing to adverse events after ICU discharge: a survey of liaison nurses. Aust Crit Care. 2013;26(2):76-80. doi: 10.1016/j.aucc.2012.07.005 pmid: 22948080

20. Yonghui W, Zongfu M, Yanru Q. Influence of music therapy on anxiety, depression and pain of cancer patients. Chin Nurs Res. 2009;13:1172-5.

21. Tahan M, Akherati Evari M, Ahangri E. The Effect of Music Therapy on Stress, Anxiety, and Depression in Patients with Cancer in Valiasr Hospital in Birjand, 2017. [Persian].J Clin Nurs Midwife. 2018;7(3):186-93.

22. Jasemi M, Aazami S, Zabihi RE. The Effects of Music Therapy on Anxiety and Depression of Cancer Patients. Indian J Palliat Care. 2016;22(4):455-8. doi: 10.4103/0973-1075.191823 pmid: 27803568

23. Baikie KA, Wilhelm K, Johnson B, Boskovic M, Wedgwood L, Finch A, et al. Expressive writing for highrisk drug dependent patients in a primary care clinic: a pilot study. Harm Reduct J. 2006;3:34. doi: 10.1186/1477-7517-3-34 pmid: 17112389

24. Petrie KJ, Fontanilla I, Thomas MG, Booth RJ, Pennebaker JW. Effect of written emotional expression on immune function in patients with human immunodeficiency virus infection: a randomized trial. Psychosom Med. 2004;66(2):272-5. doi: 10.1097/01.psy.0000116782.49850.d3 pmid: 15039514
25. Fallahi Khoshknab M, Alihasanzadeh P, Norozi K. Impacts of emotional catharsis by writing on depression in multiple sclerosis patients. [Persian]. IJPN. 2013;1(2):11-20.

26. Mosher CE, Duhamel KN, Lam J, Dickler M, Li Y, Massie $\mathrm{MJ}$, et al. Randomised trial of expressive writing for distressed metastatic breast cancer patients. Psychol Health. 2012;27(1):88-100. doi: 10.1080/08870446.2010.551212 pmid: 21678181

27. Goodman M, Yarbro CH, Frogge MH. Cancer Nursing: Principles and Practice. London. Jones2000.

28. Farokhzad P, Jannesaran M, Norouzi A, Arabloo J. Effect of Emotional Catharsis by Writing on Depression in female Cancer Patients. [Persian]. RJMS. 2017;24(156):64-71.

29. Hasanzadeh P, Fallahi Khoshknab M, Norozi K. Impacts of Journaling on Anxiety and stress in Multiple Sclerosis patients.[Persian]. Complement Med J. 2012;2(2):18393.

30. Anderson LM. Environmental genotoxicants/carcinogens and childhood cancer: filling knowledge gaps. Curr Probl Pediatr Adolesc Health Care. 2008;38(2):50-63. doi: 10.1016/j.cppeds.2007.11.002 pmid: 18237856

31. Sloan DM, Marx BP. Exposure through written emotional disclosure: Two case examples. Cognitive Behav Pract. 2006;13(3):227-34. doi: 10.1016/j.cbpra.2005.08.001

32. Delavar A. Research methodology in psychology and education. [Persian]. Tehran: Virayesh; 2015.

33. Spence SH. A measure of anxiety symptoms among children. Behave Res Ther. 1998;36(5):545-66. doi: 10.1016/S0005-7967(98)00034-5

34. Ebadinejad Z, Dashtgard A, Mirsaydy H. Effectiveness of storytelling in group discussion on anxiety of preschool children. [Persian]. JPEN. 2019;5(3):68-73.

35. Birleson P. The validity of depressive disorder in childhood and the development of a self-rating scale: a research report. J Child Psychol Psychiatry. 1981;22(1):73-88. doi: $\quad 10.1111 /$ j.14697610.1981.tb00533.x pmid: 7451588

36. Mokhtarnia I, Habibi M, Kholghi H, Mohammadi E, Kalantari F. The Study of psychometric properties of the self-rating depression scale for children and adolescents. [Persian]. Rooyesh-e-Ravanshenasi. 2018;7(4):1-22.

37. Mehrabizadeh Honarmand M, Salehi M, Kazemi N. The Effectiveness of Music Therapy and Relaxation on Blood Pressure and Pulse in the Elderly with Hypertension. [Persian]. Aging Psychol. 2017;2(4):293-303.

38. Gellaitry G, Peters K, Bloomfield D, Horne R. Narrowing the gap: the effects of an expressive writing intervention on perceptions of actual and ideal emotional support in women who have completed treatment for early stage breast cancer. Psychooncology. 2010;19(1):77-84. doi: 10.1002/pon.1532 pmid: 19194996

39. Asgari M, Makvandi B, Naderi F. The effectiveness of music therapy on anxiety and self-efficacy in the elderly [Persian]. Health Psychol. 2015;4(1):84-97. 
40. Jensen-Johansen MB, Christensen S, Valdimarsdottir H, Zakowski S, Jensen AB, Bovbjerg DH, et al. Effects of an expressive writing intervention on cancer-related distress in Danish breast cancer survivors - results from a nationwide randomized clinical trial. Psychooncology. 2013;22(7):1492-500. doi: 10.1002/pon.3193 pmid: 22991162

41. Esterling BA, L'Abate L, Murray EJ, Pennebaker JW. Empirical foundations for writing in prevention and psychotherapy: Mental and physical health outcomes. Clinic Psychol Rev. 1999;19(1):79-96. doi: 10.1016/S0272-7358(98)00015-4

42. Pennebaker JW, Chung CK. Expressive writing: Connections to physical and mental health. Oxford Handbook Health Psychol 2011:417-37. doi: 10.1093/oxfordhb/9780195342819.013.0018

43. van Emmerik AA, Reijntjes A, Kamphuis JH. Writing therapy for posttraumatic stress: a meta-analysis. Psychother Psychosom. 2013;82(2):82-8. doi: 10.1159/000343131 pmid: 23295550
44. Kross E, Duckworth A, Ayduk O, Tsukayama E, Mischel $\mathrm{W}$. The effect of self-distancing on adaptive versus maladaptive self-reflection in children. Emotion. 2011;11(5):1032-9. doi: 10.1037/a0021787 pmid: 21728415

45. Issazadegan A, Mikaeeli Manee F, Hosseinzade L. Effectiveness of Written Emotional Disclosure in Symptoms of Depression, Hope Level in Adolescents Exposed to Trauma. [Persian]. JMPR. 2014;9(1):125-41.

46. Gortner EM, Rude SS, Pennebaker JW. Benefits of expressive writing in lowering rumination and depressive symptoms. Behav Ther. 2006;37(3):292-303. doi: 10.1016/j.beth.2006.01.004 pmid: 16942980

47. Warth M, Kessler J, Koenig J, Wormit AF, Hillecke TK, Bardenheuer HJ. Music therapy to promote psychological and physiological relaxation in palliative care patients: protocol of a randomized controlled trial. BMC Palliat Care. 2014;13(1):60. doi: 10.1186/1472-684X-13-60 pmid: 25587239 